\title{
Norm of a Volterra Integral Operator on Some Analytic Function Spaces
}

\author{
Hao $\mathrm{Li}^{1}$ and Songxiao $\mathrm{Li}^{2}$ \\ ${ }^{1}$ College of Mathematics and Information Science, Henan Normal University, Xinxiang 453007, China \\ ${ }^{2}$ Department of Mathematics, Jiaying University, Meizhou, Guangdong 514015, China
}

Correspondence should be addressed to Songxiao Li; jyulsx@163.com

Received 27 June 2013; Accepted 30 July 2013

Academic Editors: O. Miyagaki and M. Winter

Copyright (c) $2013 \mathrm{H}$. Li and S. Li. This is an open access article distributed under the Creative Commons Attribution License, which permits unrestricted use, distribution, and reproduction in any medium, provided the original work is properly cited.

Let $f$ be an analytic function in the unit disc $\mathbb{D}$. The Volterra integral operator $I_{f}$ is defined as follows: $I_{f}(h)(z)=$ $\int_{0}^{z} f(w) h^{\prime}(w) d w, h \in H(\mathbb{D}), z \in \mathbb{D}$. In this paper, we compute the norm of $I_{f}$ on some analytic function spaces.

\section{Introduction}

Let $\mathbb{D}=\{z:|z|<1\}$ be the unit disk of complex plane $\mathbb{C}$ and $H(\mathbb{D})$ the class of functions analytic in $\mathbb{D}$. Denote by $d \sigma$ the normalized Lebesgue area measure in $\mathbb{D}$ and $g(a, z)$ the Green function with logarithmic singularity at $a$; that is, $g(a, z)=$ $-\log \left|\varphi_{a}(z)\right|$, where $\varphi_{a}(z)=(a-z) /(1-\bar{a} z)$ is the Möbius transformation of $\mathbb{D}$.

Let $0<p<\infty$. The $Q_{p}$ is the space of all functions $f \in$ $H(\mathbb{D})$ such that

$$
\begin{aligned}
\|f\|_{Q_{p}}^{2}= & |f(0)|^{2} \\
& +\sup _{a \in \mathbb{D}} \int_{\mathbb{D}}\left|f^{\prime}(z)\right|^{2}\left(1-\left|\varphi_{a}(z)\right|^{2}\right)^{p} d \sigma(z)<\infty .
\end{aligned}
$$

From $[1,2]$, we see that $Q_{1}=$ BMOA, the space of all analytic functions of bounded mean oscillation. When $p>1$, the space $Q_{p}$ is the same and equal to the Bloch space $\mathfrak{B}$, which consists of all $f \in H(\mathbb{D})$ for which

$$
\|f\|_{\mathfrak{B}}=|f(0)|+\sup _{z \in \mathbb{D}}\left|f^{\prime}(z)\right|\left(1-|z|^{2}\right)<\infty .
$$

See $[3,4]$ for the theory of Bloch functions.
For $\alpha>0$, the $\alpha$-Bloch space, denoted by $\mathfrak{B}^{\alpha}$, is the space of all $f \in H(\mathbb{D})$ such that

$$
\|f\|_{\mathfrak{B}^{\alpha}}=|f(0)|+\sup _{z \in \mathbb{D}}\left|f^{\prime}(z)\right|\left(1-|z|^{2}\right)^{\alpha}<\infty .
$$

It is clear that $\mathfrak{B}^{\alpha_{1}} \varsubsetneqq \mathfrak{B} \varsubsetneqq \mathfrak{B}^{\alpha_{2}}$ for $0<\alpha_{1}<1<\alpha_{2}<\infty$.

Let $1 \leqslant q \leqslant \infty$ and let $0 \leqslant \alpha \leqslant 1$. The mean Lipschitz space $\Lambda(q, \alpha)$ consists of those functions $f \in H(\mathbb{D})$ for which

$$
\begin{aligned}
\|f\|_{\Lambda(q, \alpha)}= & |f(0)|+\sup _{0 \leqslant r<1}\left(1-r^{2}\right)^{1-\alpha} \\
& \times\left(\frac{1}{2 \pi} \int_{0}^{2 \pi}\left|f^{\prime}\left(r e^{i \varphi}\right)\right|^{q} d \varphi\right)^{1 / q}<\infty .
\end{aligned}
$$

It is obvious that $\Lambda(\infty, 0)$ is just the Bloch space $\mathfrak{B}$, which is contained in $\Lambda(q, 0)$ for every $1<q<\infty$. Note that $\Lambda(q, 1 / q)$ increases with $q \in(1, \infty)$. We refer to [5] for more information of mean Lipschitz spaces.

For $0 \leqslant s<\infty$, we say that an $f \in H(\mathbb{D})$ belongs to the growth space $H_{s}^{\infty}$ if

$$
\|f\|_{H_{s}^{\infty}}=\sup _{0 \leqslant r<1}\left|f\left(r e^{i \theta}\right)\right|\left(1-r^{2}\right)^{s}<\infty .
$$

It is easy to see that $H_{0}^{\infty}=H^{\infty}$. 
For $-1<\alpha<\infty$, an $f \in H(\mathbb{D})$ is said to belong to the $\mathscr{D}^{\alpha}$ space if

$$
\|f\|_{\mathscr{D}^{\alpha}}^{2}=|f(0)|^{2}+\int_{\mathbb{D}}\left|f^{\prime}(z)\right|^{2}\left(1-|z|^{2}\right)^{\alpha} d \sigma(z)<\infty .
$$

For $1<p<\infty$, the Besov space $\mathscr{B}_{p}$ is defined to be the space of all analytic functions $f$ in $\mathbb{D}$ such that

$$
\|f\|_{\mathscr{B}_{p}}^{p}=|f(0)|^{p}+\int_{\mathbb{D}}\left|f^{\prime}(z)\right|^{p}\left(1-|z|^{2}\right)^{p-2} d \sigma(z)<\infty .
$$

Let $f \in H(\mathbb{D})$. The Volterra integral operators $I_{f}$ and $J_{f}$ are defined as follows:

$$
\begin{array}{r}
I_{f}(h)(z)=\int_{0}^{z} h^{\prime}(w) f(w) d w, \\
J_{f}(h)(z)=\int_{0}^{z} h(w) f^{\prime}(w) d w, \\
(z \in \mathbb{D}) .
\end{array}
$$

It is easy to see that

$$
\left(I_{f}+J_{f}\right) h+f(0) h(0)=M_{f}(h),
$$

where $M_{f}$ denotes the multiplication operator; that is, $M_{f}(h)=f h$. If $f$ is a constant, then all results about $I_{f}$, $J_{f}$, or $M_{f}$ are trivial. In this paper, we assume that $f$ is a nonconstant. Both operators have been studied by many authors. See [6-23] and the references therein.

Norms of some special operators, such as composition operator, weighted composition operator, and some integral operators, have been studied by many authors. The interested readers can refer [13,24-32], for example. Recently, Liu and Xiong studied the norm of integral operators $I_{f}$ and $J_{f}$ on the Bloch space, Dirichlet space, BMOA space, and so on in [13]. In this paper, we study the norm of integral operator $I_{f}$ on some function spaces in the unit disk.

\section{Main Results}

In this section, we state and prove our main results. In order to formulate our main results, we need some auxiliary results which are incorporated in the following lemmas.

Lemma 1 (see [5, page 144]). If $f \in H^{p}(0<p \leqslant \infty)$, then $|f(z)| \leqslant\left(1-|z|^{2}\right)^{-1 / p}\|f\|_{p},|z|<1$, and the inequality is sharp for each fixed $z$.

Lemma 2. Let $-1<\alpha<\infty$ and $0<p<\infty$. For any $f \in$ $H(\mathbb{D})$, the following one has:

$$
|f(a)|^{p} \leqslant(\alpha+1) \int_{\mathbb{D}}|f(z)|^{p} \frac{\left(1-|a|^{2}\right)^{2+\alpha}}{|1-\bar{a} z|^{4+2 \alpha}}\left(1-|z|^{2}\right)^{\alpha} d \sigma(z),
$$

where $a$ is any point in $\mathbb{D}$.
Proof. For any $f \in H(\mathbb{D})$, taking $z=r e^{i \theta}$ and the subharmonicity of $|f(z)|^{p}$, we get

$$
|f(0)|^{p} \leqslant \frac{1}{2 \pi} \int_{0}^{2 \pi}\left|f\left(r e^{i \theta}\right)\right|^{p} d \theta
$$

and so

$$
\begin{aligned}
|f(0)|^{p} \leqslant(\alpha+1) \frac{1}{\pi} \int_{0}^{1} \int_{0}^{2 \pi}\left|f\left(r e^{i \theta}\right)\right|^{p} & \times\left(1-r^{2}\right)^{\alpha} d \theta r d r \\
= & (\alpha+1) \int_{\mathbb{D}}|f(z)|^{p}\left(1-|z|^{2}\right)^{\alpha} d \sigma(z) .
\end{aligned}
$$

For any $a \in \mathbb{D}$, let $\varphi_{a}(z)=(a-z) /(1-\bar{a} z)$. Replacing $f$ by $f \circ \varphi_{a}(z)$ and applying the change of variable formula give the following:

$$
\begin{aligned}
|f(a)|^{p} & \leqslant(\alpha+1) \int_{\mathbb{D}}\left|f \circ \varphi_{a}(z)\right|^{p}\left(1-|z|^{2}\right)^{\alpha} d \sigma(z) \\
& =(\alpha+1) \int_{\mathbb{D}}|f(z)|^{p}\left(1-\left|\varphi_{a}(z)\right|^{2}\right)^{\alpha} \frac{\left(1-|a|^{2}\right)^{2}}{|1-\bar{a} z|^{4}} d \sigma(z) \\
& =(\alpha+1) \int_{\mathbb{D}}|f(z)|^{p}\left(1-|a|^{2}\right)^{2+\alpha} \frac{\left(1-|z|^{2}\right)^{\alpha}}{|1-\bar{a} z|^{2 \alpha+4}} d \sigma(z) .
\end{aligned}
$$

The proof is complete.

Theorem 3. Let $f \in H(\mathbb{D})$. The integral operator $I_{f}$ is bounded on $\Lambda(1,1)$ if and only if $f \in H^{\infty}$. Moreover, one has

$$
\left\|I_{f}\right\|=\|f\|_{H^{\infty}} .
$$

Proof. If $f \in H^{\infty}$, by (4), we have

$$
\begin{aligned}
\left\|I_{f} h\right\|_{\Lambda(1,1)} & =\sup _{0 \leqslant r<1}\left(\frac{1}{2 \pi} \int_{0}^{2 \pi}\left|f\left(r e^{i \varphi}\right) h^{\prime}\left(r e^{i \varphi}\right)\right| d \varphi\right) \\
& \leqslant\|f\|_{H^{\infty}} \sup _{0 \leqslant r<1}\left(\frac{1}{2 \pi} \int_{0}^{2 \pi}\left|h^{\prime}\left(r e^{i \varphi}\right)\right| d \varphi\right) \\
& \leqslant\|f\|_{H^{\infty}}\|h\|_{\Lambda(1,1)} .
\end{aligned}
$$

Thus $\left\|I_{f}\right\| \leqslant\|f\|_{H^{\infty}}$.

On the other hand, denote $c=\sup _{z \in \mathbb{D}}|f(z)|$. Given any $\epsilon>0$, there exists $z_{1} \in \mathbb{D}$ such that $\left|f\left(z_{1}\right)\right|>c-\epsilon$. Let

$$
h(z)=\frac{z_{1}-z}{1-\bar{z}_{1} z}-z_{1} .
$$


Then we have $\|h\|_{\Lambda(1,1)}=1$. In fact, taking $z_{1}=r_{1} e^{i \varphi_{1}}$ and $z=r e^{i \varphi}$ and using Poisson integral, we get

$$
\begin{aligned}
\|h\|_{\Lambda(1,1)} & =\sup _{0 \leqslant r<1}\left(\frac{1}{2 \pi} \int_{0}^{2 \pi}\left|h^{\prime}\left(r e^{i \varphi}\right)\right| d \varphi\right) \\
& =\sup _{0 \leqslant r<1}\left(\frac{1}{2 \pi} \int_{0}^{2 \pi} \frac{1-r_{1}^{2}}{\left|1-r r_{1} e^{i\left(\varphi-\varphi_{1}\right)}\right|^{2}} d \varphi\right) \\
& =\sup _{0 \leqslant r<1} \frac{1-r_{1}^{2}}{1-r^{2} r_{1}^{2}}=1 .
\end{aligned}
$$

Taking $z_{1}=r_{1} e^{i \varphi_{1}}$, we obtain

$$
\begin{aligned}
\infty>\left\|I_{f}\right\| \geqslant\left\|I_{f} h\right\|_{\Lambda(1,1)} \\
=\sup _{0 \leqslant r<1}\left(\frac{1}{2 \pi} \int_{0}^{2 \pi}\left|f\left(r e^{i \varphi}\right) h^{\prime}\left(r e^{i \varphi}\right)\right| d \varphi\right) \\
=\sup _{0 \leqslant r<1}\left(\frac{1}{2 \pi} \int_{0}^{2 \pi}\left|f\left(r e^{i \varphi}\right)\right|\right. \\
\left.\quad \times \frac{1-r_{1}^{2}}{\left|1-r_{1} r e^{i\left(\varphi-\varphi_{1}\right)}\right|^{2}} d \varphi\right) .
\end{aligned}
$$

So $f h^{\prime} \in H^{1}$ and $f \in H^{1}$. Thus Theorem 2.6 in [5] yields

$$
\left\|I_{f}\right\| \geqslant\left(\frac{1}{2 \pi} \int_{0}^{2 \pi}\left|f\left(e^{i \varphi}\right)\right| \frac{1-r_{1}^{2}}{\left|1-r_{1} e^{i\left(\varphi-\varphi_{1}\right)}\right|^{2}} d \varphi\right) .
$$

By Theorem 2.12 in [5], we have

$$
\left\|I_{f}\right\| \geqslant\left|f\left(z_{1}\right)\right|>c-\epsilon,
$$

so the arbitrariness of $\epsilon$ gives $\left\|I_{f}\right\| \geqslant\|f\|_{H^{\infty}}$ and the proof is complete.

Lemma 3 in [13] gives the norm of $I_{f}$ on Dirichlet space. Here, we consider the norm of $I_{f}$ on $\alpha$-Dirichlet space $\mathscr{D}^{\alpha}$.

Theorem 4. Let $f \in H(\mathbb{D})$ and $-1<\alpha<\infty$. Then $I_{f}$ is bounded on $\mathscr{D}^{\alpha}$ if and only if $f \in H^{\infty}$. Moreover, one has

$$
\left\|I_{f}\right\|=\|f\|_{H^{\infty}}
$$

Proof. First, we assume that $f \in H^{\infty}$. Let $h \in \mathscr{D}^{\alpha}$. Then (6) gives

$$
\begin{aligned}
\left\|I_{f} h\right\|_{\mathscr{D}^{\alpha}}^{2} & =\int_{\mathbb{D}}\left|f(z) h^{\prime}(z)\right|^{2}\left(1-|z|^{2}\right)^{\alpha} d \sigma(z) \\
& \leqslant\|f\|_{H^{\infty}}^{2} \int_{\mathbb{D}}\left|h^{\prime}(z)\right|^{2}\left(1-|z|^{2}\right)^{\alpha} d \sigma(z) \\
& \leqslant\|f\|_{H^{\infty}}^{2}\|h\|_{D^{\alpha}}^{2},
\end{aligned}
$$

and so we have $\left\|I_{f}\right\| \leqslant\|f\|_{H^{\infty}}$.
Now we need only to show the reverse inequality. Denote $c=\sup _{z \in \mathbb{D}}|f(z)|$. Given any $\epsilon>0$, there exists $z_{1} \in \mathbb{D}$ such that $\left|f\left(z_{1}\right)\right|>c-\epsilon$. Let

$$
h_{1}(z)=\int_{\Gamma(z)} \frac{\left(1-\left|z_{1}\right|^{2}\right)^{1+(\alpha / 2)}}{\left(1-\bar{z}_{1} \zeta\right)^{2+\alpha}} d \zeta
$$

where $\Gamma(z)$ is any path in $\mathbb{D}$ from 0 to $z$. By Theorem 13.11 in [33, page 274], we know $h_{1}$ is an analytic function in $\mathbb{D}$ and $h_{1}^{\prime}(z)=\left(1-\left|z_{1}\right|^{2}\right)^{1+(\alpha / 2)} /\left(1-\bar{z}_{1} z\right)^{2+\alpha}$. Also it is easy to check that $\left\|h_{1}\right\|_{\mathscr{D}^{\alpha}}^{2}=1 /(\alpha+1)$. Indeed, by using the method of the proof of Lemma 4.2.2 in [4], we have

$$
\begin{aligned}
\left\|h_{1}\right\|_{\mathscr{D}^{\alpha}}^{2} & =\int_{\mathbb{D}} \frac{\left(1-\left|z_{1}\right|^{2}\right)^{2+\alpha}}{\left|1-\bar{z}_{1} z\right|^{4+2 \alpha}}\left(1-|z|^{2}\right)^{\alpha} d \sigma(z) \\
& =\left(1-\left|z_{1}\right|^{2}\right)^{2+\alpha} \int_{\mathbb{D}} \frac{\left(1-|z|^{2}\right)^{\alpha}}{\left|1-\bar{z}_{1} z\right|^{4+2 \alpha}} d \sigma(z) \\
& =\left(1-\left|z_{1}\right|^{2}\right)^{2+\alpha} \frac{\Gamma(\alpha+1)}{\Gamma^{2}(\alpha+2)} \sum_{n=0}^{\infty} \frac{\Gamma^{2}(n+2+\alpha)}{n ! \Gamma(n+2+\alpha)}\left|z_{1}\right|^{2 n} \\
& =\frac{\left(1-\left|z_{1}\right|^{2}\right)^{2+\alpha}}{\alpha+1} \sum_{n=0}^{\infty} \frac{\Gamma(n+2+\alpha)}{n ! \Gamma(\alpha+2)}\left|z_{1}\right|^{2 n} \\
& =\frac{\left(1-\left|z_{1}\right|^{2}\right)^{2+\alpha}}{\alpha+1} \frac{1}{\left(1-\left|z_{1}\right|^{2}\right)^{2+\alpha}}=\frac{1}{\alpha+1} .
\end{aligned}
$$

Let $h(z)=h_{1}(z) /\left\|h_{1}\right\|_{\mathscr{D}^{\alpha}}$, and so $\|h\|_{\mathscr{D}^{\alpha}}=1$. Thus by Lemma 2 we have

$$
\begin{aligned}
\left\|I_{f}\right\|^{2} & \geq\left\|I_{f} h(z)\right\|_{D^{\alpha}}^{2} \\
& =\int_{\mathbb{D}}\left|f(z) h^{\prime}(z)\right|^{2}\left(1-|z|^{2}\right)^{\alpha} d \sigma(z) \\
& =(\alpha+1) \int_{\mathbb{D}}|f(z)|^{2} \frac{\left(1-\left|z_{1}\right|^{2}\right)^{2+\alpha}}{\left|1-\bar{z}_{1} z\right|^{4+2 \alpha}}\left(1-|z|^{2}\right)^{\alpha} d \sigma(z) \\
& \geqslant\left|f\left(z_{1}\right)\right|^{2}>(c-\epsilon)^{2} .
\end{aligned}
$$

Since $\epsilon$ is arbitrary, we get

$$
\left\|I_{f}\right\| \geqslant\|f\|_{H^{\infty}}
$$

which implies the desired result.

Theorem 5. Let $f \in H(\mathbb{D})$ and let $1<p<\infty$. The integral operator $I_{f}$ is bounded on $\mathscr{B}_{p}$ if and only if $f \in H^{\infty}$. Moreover, one has

$$
\left\|I_{f}\right\|=\|f\|_{H^{\infty}}
$$


Proof. If $f \in H^{\infty}$, then by (7), we have

$$
\begin{aligned}
\left\|I_{f} h\right\|_{\mathscr{B}_{p}}^{p} & =\int_{\mathbb{D}}\left|f(z) h^{\prime}(z)\right|^{p}\left(1-|z|^{2}\right)^{p-2} d \sigma(z) \\
& \leqslant\|f\|_{H^{\infty}}^{p} \int_{\mathbb{D}}\left|h^{\prime}(z)\right|^{p}\left(1-|z|^{2}\right)^{p-2} d \sigma(z) \\
& \leqslant\|f\|_{H^{\infty}}^{p}\|h\|_{\mathscr{B}_{p}}^{p},
\end{aligned}
$$

and so $\left\|I_{f}\right\| \leqslant\|f\|_{H^{\infty}}$.

Now we need only to show the reverse inequality. Denote $c=\sup _{z \in \mathbb{D}}|f(z)|$. Given any $\epsilon>0$, there exists $z_{1} \in \mathbb{D}$ such that $\left|f\left(z_{1}\right)\right|>c-\epsilon$. Let

$$
h_{1}(z)=\frac{z_{1}-z}{1-z_{1} z}-z_{1}, \quad z \in \mathbb{D} .
$$

We see that $\left\|h_{1}\right\|_{\mathscr{B}_{p}}^{p}=1 /(p-1)$. Indeed,

$$
\begin{aligned}
\left\|h_{1}\right\|_{\mathscr{B}_{p}}^{p} & =\int_{\mathbb{D}} \frac{\left(1-\left|z_{1}\right|^{2}\right)^{p}}{\left|1-\bar{z}_{1} z\right|^{2 p}}\left(1-|z|^{2}\right)^{p-2} d \sigma(z) \\
& =\left(1-\left|z_{1}\right|^{2}\right)^{p} \int_{\mathbb{D}} \frac{\left(1-|z|^{2}\right)^{p-2}}{\left|1-\bar{z}_{1} z\right|^{2 p}} d \sigma(z) \\
& =\left(1-\left|z_{1}\right|^{2}\right)^{p} \frac{\Gamma(p-1)}{\Gamma^{2}(p)} \sum_{n=0}^{\infty} \frac{\Gamma(n+p)^{2}}{n ! \Gamma(n+p)}\left|z_{1}\right|^{2 n} \\
& =\frac{\left(1-\left|z_{1}\right|^{2}\right)^{p}}{p-1} \sum_{n=0}^{\infty} \frac{\Gamma(n+p)}{n ! \Gamma(p)}\left|z_{1}\right|^{2 n} \\
& =\frac{\left(1-\left|z_{1}\right|^{2}\right)^{p}}{p-1} \frac{1}{\left(1-\left|z_{1}\right|^{2}\right)^{p}}=\frac{1}{p-1} .
\end{aligned}
$$

Let $h(z)=h_{1}(z) /\left\|h_{1}\right\|_{\mathscr{B}_{p}}$. Then $\|h\|_{\mathscr{B}_{p}}=1$. Thus by Lemma 2, we have

$$
\begin{aligned}
\left\|I_{f}\right\|^{p} \geq\left\|I_{f} h(z)\right\|_{\mathscr{B}_{p}}^{p} & =\int_{\mathbb{D}}\left|f(z) h^{\prime}(z)\right|^{p}\left(1-|z|^{2}\right)^{p-2} d \sigma(z) \\
& =(p-1) \int_{\mathbb{D}}|f(z)|^{p} \frac{\left(1-\left|z_{1}\right|^{2}\right)^{p}}{\left|1-\bar{z}_{1} z\right|^{2 p}} \\
& \times\left(1-|z|^{2}\right)^{p-2} d \sigma(z) \\
\geqslant & \left|f\left(z_{1}\right)\right|^{p}>(c-\epsilon)^{p} .
\end{aligned}
$$

Since $\epsilon$ is arbitrary, we get $\left\|I_{f}\right\| \geqslant\|f\|_{H^{\infty}}$. The proof is complete.

Theorem 6. Let $f \in H(\mathbb{D})$ and let $0<\alpha \leqslant \beta<\infty$. The integral operator $I_{f}$ is bounded from $\mathfrak{B}^{\alpha}$ to $\mathfrak{B}^{\beta}$ if and only if $f \in H_{\beta-\alpha}^{\infty}$. Moreover, one has

$$
\left\|I_{f}\right\|=\sup _{z \in \mathbb{D}}|f(z)|\left(1-|z|^{2}\right)^{\beta-\alpha} .
$$

Proof. If $f \in H_{\beta-\alpha}^{\infty}$, then by (3), we have

$$
\begin{aligned}
\left\|I_{f} h\right\|_{\mathfrak{B}^{\beta}} & =\sup _{z \in \mathbb{D}}\left|f(z) h^{\prime}(z)\right|\left(1-|z|^{2}\right)^{\beta} \\
& \leqslant\|f\|_{H_{\beta-\alpha}^{\infty} \sup _{z \in \mathbb{D}}}\left|h^{\prime}(z)\right|\left(1-|z|^{2}\right)^{\alpha} \\
& \leqslant\|f\|_{H_{\beta-\alpha}^{\infty}}\|h\|_{\mathcal{B}^{\alpha}} .
\end{aligned}
$$

Hence $\left\|I_{f}\right\| \leqslant\|f\|_{H_{\beta-\alpha}^{\infty}}$.

For the converse, denote $c=\sup _{z \in \mathbb{D}}|f(z)|\left(1-|z|^{2}\right)^{\beta-\alpha}$. Given any $\epsilon>0$, there exists $z_{1} \in \mathbb{D}$ such that $\left|f\left(z_{1}\right)\right|(1-$ $\left.\left|z_{1}\right|^{2}\right)^{\beta-\alpha}>c-\epsilon$. Set

$$
h(z)=\int_{\Gamma(z)} \frac{\left(1-\left|z_{1}\right|^{2}\right)^{\alpha}}{\left(1-\bar{z}_{1} \zeta\right)^{2 \alpha}} d \zeta,
$$

where $\Gamma(z)$ is any path in $\mathbb{D}$ from 0 to $z$. By Theorem 13.11 in [33, page 274], we know that $h$ is an analytic function in $\mathbb{D}$ and $h^{\prime}(z)=\left(1-\left|z_{1}\right|^{2}\right)^{\alpha} /\left(1-\bar{z}_{1} z\right)^{2 \alpha}$, and it is easy to check that $\|h\|_{\mathfrak{B}^{\alpha}}=1$. Thus

$$
\begin{aligned}
\left\|I_{f}\right\| & \geqslant\left\|I_{f} h\right\|_{\mathfrak{B}^{\beta}} \\
& =\sup _{z \in \mathbb{D}}\left|f(z) h^{\prime}(z)\right|\left(1-|z|^{2}\right)^{\beta} \\
& \geqslant\left|f\left(z_{1}\right) h^{\prime}\left(z_{1}\right)\right|\left(1-\left|z_{1}\right|^{2}\right)^{\beta} \\
& \geqslant\left|f\left(z_{1}\right)\right| \frac{\left(1-\left|z_{1}\right|^{2}\right)^{\alpha}}{\left(1-\left|z_{1}\right|^{2}\right)^{2 \alpha}}\left(1-\left|z_{1}\right|^{2}\right)^{\beta} \\
& =\left|f\left(z_{1}\right)\right|\left(1-\left|z_{1}\right|^{2}\right)^{\beta-\alpha}>c-\epsilon .
\end{aligned}
$$

Since $\epsilon$ is arbitrary, we obtain the desired result. The proof is complete.

Theorem 7. Let $f \in H(\mathbb{D})$. The integral operator $I_{f}$ is bounded from $\Lambda(1,1)$ to $\mathfrak{B}$ if and only if $f \in H^{\infty}$. Moreover, one has

$$
\left\|I_{f}\right\|=\|f\|_{H^{\infty}}
$$

Proof. If $f \in H^{\infty}$, then by Lemma 1, we have

$$
\begin{aligned}
\left\|I_{f} h\right\|_{\mathfrak{B}} & =\sup _{z \in \mathbb{D}}\left|f(z) h^{\prime}(z)\right|\left(1-|z|^{2}\right) \\
& \leqslant\|h\|_{\Lambda(1,1)} \sup _{z \in \mathbb{D}}\left(1-|z|^{2}\right)^{-1}\left(1-|z|^{2}\right)|f(z)| \\
& =\|h\|_{\Lambda(1,1)} \sup _{z \in \mathbb{D}}|f(z)|,
\end{aligned}
$$

and hence $\left\|I_{f}\right\| \leqslant\|f\|_{H^{\infty}}$

For the converse, denote $c=\sup _{z \in \mathbb{D}}|f(z)|$. Given any $\epsilon>$ 0 , there exists $z_{1} \in \mathbb{D}$ such that $\left|f\left(z_{1}\right)\right|>c-\epsilon$. Let

$$
h(z)=\frac{z_{1}-z}{1-z_{1} z}-z_{1}, \quad z \in \mathbb{D} .
$$


Then by the proof of Theorem 3, we see that $\|h\|_{\Lambda(1,1)}=1$. In the meantime, we know that $\left|h^{\prime}\left(z_{1}\right)\right|\left(1-\left|z_{1}\right|^{2}\right)=1$, which gives

$$
\begin{aligned}
\left\|I_{f}\right\| & \geqslant\left\|I_{f} h\right\|_{\mathfrak{B}} \\
& =\sup _{z \in \mathbb{D}}\left|f(z) h^{\prime}(z)\right|\left(1-|z|^{2}\right) \\
& \geqslant\left|f\left(z_{1}\right) h^{\prime}\left(z_{1}\right)\right|\left(1-\left|z_{1}\right|^{2}\right) \\
& =\left|f\left(z_{1}\right)\right|>c-\epsilon .
\end{aligned}
$$

Since $\epsilon$ is arbitrary, we get the desired result. The proof is complete.

Finally, we consider the norm of $I_{f}$ from $\Lambda(\infty, 1)$ to some Banach spaces.

Theorem 8. If $f \in H(\mathbb{D})$, then the following assertions hold.

(1) Let $0<p<\infty$. The integral operator $I_{f}$ is bounded from $\Lambda(\infty, 1)$ space to $Q_{p}$ space if and only if $f$ satisfies

$$
\sup _{a \in \mathbb{D}} \int_{\mathbb{D}}|f(z)|^{2}\left(1-\left|\varphi_{a}(z)\right|^{2}\right)^{p} d \sigma(z)<\infty
$$

Moreover, one has

$$
\left\|I_{f}\right\|=\sup _{a \in \mathbb{D}} \int_{\mathbb{D}}|f(z)|^{2}\left(1-\left|\varphi_{a}(z)\right|^{2}\right)^{p} d \sigma(z) .
$$

(2) Let $0 \leqslant \alpha \leqslant 1$ and let $0 \leqslant q \leqslant \infty$. The integral operator $I_{f}$ is bounded from $\Lambda(\infty, 1)$ space to $\Lambda(q, \alpha)$ space if and only if $f$ satisfies

$$
\sup _{0 \leqslant r<1}\left(1-r^{2}\right)^{1-\alpha}\left(\frac{1}{2 \pi} \int_{0}^{2 \pi}\left|f\left(r e^{i \varphi}\right)\right|^{q} d \varphi\right)^{1 / q}<\infty .
$$

Moreover, one has

$$
\left\|I_{f}\right\|=\sup _{0 \leqslant r<1}\left(1-r^{2}\right)^{1-\alpha}\left(\frac{1}{2 \pi} \int_{0}^{2 \pi}\left|f\left(r e^{i \varphi}\right)\right|^{q} d \varphi\right)^{1 / q} .
$$

(3) Let $0<\alpha<\infty$. The integral operator $I_{f}$ is bounded from $\Lambda(\infty, 1)$ space to $\mathfrak{B}^{\alpha}$ space if and only if $f$ satisfies $\sup _{z \in \mathbb{D}}|f(z)|\left(1-|z|^{2}\right)^{\alpha}<\infty$. Moreover, one has

$$
\left\|I_{f}\right\|=\sup _{z \in \mathbb{D}}|f(z)|\left(1-|z|^{2}\right)^{\alpha}
$$

(4) Let $-1<\alpha<\infty$. The integral operator $I_{f}$ is bounded from $\Lambda(\infty, 1)$ space to $\mathscr{D}^{\alpha}$ space if and only if $f$ satisfies $\int_{\mathbb{D}}|f(z)|^{2}\left(1-|z|^{2}\right)^{\alpha} d \sigma(z)<\infty$. Moreover, one has

$$
\left\|I_{f}\right\|=\int_{\mathbb{D}}|f(z)|^{2}\left(1-|z|^{2}\right)^{\alpha} d \sigma(z) .
$$

Proof. The assertion (1) will be proved only here, and the conclusions of (2), (3), and (4) follow by using the similar arguments to that used in proving (1), and so the proofs are omitted.

If $h \in \Lambda(\infty, 1)$, then by (1), we have

$$
\begin{aligned}
\left\|I_{f} h\right\|_{Q_{p}}^{2} & =\sup _{a \in \mathbb{D}} \int_{\mathbb{D}}\left|f(z) h^{\prime}(z)\right|^{2}\left(1-\left|\varphi_{a}(z)\right|^{2}\right)^{p} d \sigma(z) \\
& \leqslant\|h\|_{\Lambda(\infty, 1)}^{2} \sup _{a \in \mathbb{D}} \int_{\mathbb{D}}|f(z)|^{2}\left(1-\left|\varphi_{a}(z)\right|^{2}\right)^{p} d \sigma(z),
\end{aligned}
$$

and so

$$
\left\|I_{f}\right\| \leqslant \sup _{a \in \mathbb{D}} \int_{\mathbb{D}}|f(z)|^{2}\left(1-\left|\varphi_{a}(z)\right|^{2}\right)^{p} d \sigma(z) .
$$

For the converse, let $h(z)=z$. It is easy to see that $\|h\|_{\Lambda(\infty, 1)}=1$. Thus

$$
\begin{aligned}
\left\|I_{f}\right\| & \geqslant\left\|I_{f} h\right\|_{Q_{p}}^{2} \\
& =\sup _{a \in \mathbb{D}} \int_{\mathbb{D}}\left|f(z) h^{\prime}(z)\right|^{2}\left(1-\left|\varphi_{a}(z)\right|^{2}\right)^{p} d \sigma(z) \\
& =\sup _{a \in \mathbb{D}} \int_{\mathbb{D}}|f(z)|^{2}\left(1-\left|\varphi_{a}(z)\right|^{2}\right)^{p} d \sigma(z) .
\end{aligned}
$$

The desired result follows by (47) and (48). The proof is complete.

\section{Acknowledgments}

Hao Li is supported by the National Natural Science Foundation of China (no. 11126284). Songxiao Li is supported by the project of Department of Education of Guangdong Province (no. 2012KJCX0096).

\section{References}

[1] R. Aulaskari, J. Xiao, and R. H. Zhao, "On subspaces and subsets of BMOA and UBC," Analysis, vol. 15, no. 2, pp. 101-121, 1995.

[2] J. Xiao, Holomorphic Q Classes, vol. 1767 of Lecture Notes in Mathematics, Springer, Berlin, Germany, 2001.

[3] K. H. Zhu, "Bloch type spaces of analytic functions," The Rocky Mountain Journal of Mathematics, vol. 23, no. 3, pp. 1143-1177, 1993.

[4] K. H. Zhu, Operator Theory in Function Spaces, vol. 139 of Monographs and Textbooks in Pure and Applied Mathematics, Marcel Dekker, New York, NY, USA, 1990.

[5] P. L. Duren, Theory of $H^{p}$ Spaces, vol. 38 of Pure and Applied Mathematics, Academic Press, New York, NY, USA, 1970.

[6] A. Aleman and A. G. Siskakis, "An integral operator on $H^{p}$," Complex Variables, vol. 28, no. 2, pp. 149-158, 1995.

[7] A. Aleman and A. G. Siskakis, "Integration operators on Bergman spaces," Indiana University Mathematics Journal, vol. 46, no. 2, pp. 337-356, 1997.

[8] A. Austin, Multiplication and integral operators on Banach spaces of analytic functions [Ph.D. thesis], University of Hawai, 2010. 
[9] S. Li and S. Stević, "Volterra-type operators on Zygmund spaces," Journal of Inequalities and Applications, vol. 2007, Article ID 32124, 10 pages, 2007.

[10] S. Li and S. Stević, "Generalized composition operators on Zygmund spaces and Bloch type spaces," Journal of Mathematical Analysis and Applications, vol. 338, no. 2, pp. 1282-1295, 2008.

[11] S. Li and S. Stević, "Products of integral-type operators and composition operators between Bloch-type spaces," Journal of Mathematical Analysis and Applications, vol. 349, no. 2, pp. 596610, 2009.

[12] J. Liu, Z. Lou, and C. Xiong, "Essential norms of integral operators on spaces of analytic functions," Nonlinear Analysis: Theory, Methods \& Applications, vol. 75, no. 13, pp. 5145-5156, 2012.

[13] J. Liu and C. Xiong, "Norm-attaining integral operators on analytic function spaces," Journal of Mathematical Analysis and Applications, vol. 399, no. 1, pp. 108-115, 2013.

[14] C. Pan, "On an integral-type operator from $Q_{K}(p, q)$ spaces to $\alpha$-Bloch spaces," Filomat, vol. 25, no. 3, pp. 163-173, 2011.

[15] Ch. Pommerenke, "Schlichte Funktionen und analytische Funktionen von beschränkter mittlerer Oszillation," Commentarii Mathematici Helvetici, vol. 52, no. 4, pp. 591-602, 1977.

[16] S. Stević, "Generalized composition operators between mixednorm and some weighted spaces," Numerical Functional Analysis and Optimization, vol. 29, no. 7-8, pp. 959-978, 2008.

[17] A. G. Siskakis and R. Zhao, "A Volterra type operator on spaces of analytic functions," in Function Spaces (Edwardsville, IL, 1998), vol. 232 of Contemp. Math., pp. 299-311, American Mathematical Society, Providence, RI, USA, 1999.

[18] S. Stević, "On an integral operator between Bloch-type spaces on the unit ball," Bulletin des Sciences Mathématiques, vol. 134, no. 4, pp. 329-339, 2010.

[19] W. Yang, "On an integral-type operator between Bloch-type spaces," Applied Mathematics and Computation, vol. 215, no. 3, pp. 954-960, 2009.

[20] X. Zhu, "Generalized composition operators from generalized weighted Bergman spaces to Bloch type spaces," Journal of the Korean Mathematical Society, vol. 46, no. 6, pp. 1219-1232, 2009.

[21] X. Zhu, "Generalized composition operators and Volterra composition operators on Bloch spaces in the unit ball," Complex Variables and Elliptic Equations, vol. 54, no. 2, pp. 95-102, 2009.

[22] X. Zhu, "An integral-type operator from $H^{\infty}$ to Zygmund-type spaces," Bulletin of the Malaysian Mathematical Sciences Society, vol. 35, no. 3, pp. 679-686, 2012.

[23] S. Li, "On an integral-type operator from the Bloch space into the $Q_{K}(p, q)$ space," Filomat, vol. 26, pp. 125-133, 2012.

[24] P. S. Bourdon, E. E. Fry, C. Hammond, and C. H. Spofford, "Norms of linear-fractional composition operators," Transactions of the American Mathematical Society, vol. 356, no. 6, pp. 2459-2480, 2004.

[25] F. Colonna, G. R. Easley, and D. Singman, "Norm of the multiplication operators from $H^{\infty}$ to the Bloch space of a bounded symmetric domain," Journal of Mathematical Analysis and Applications, vol. 382, no. 2, pp. 621-630, 2011.

[26] C. Hammond, "The norm of a composition operator with linear symbol acting on the Dirichlet space," Journal of Mathematical Analysis and Applications, vol. 303, no. 2, pp. 499-508, 2005.

[27] M. J. Martín, "Norm-attaining composition operators on the Bloch spaces," Journal of Mathematical Analysis and Applications, vol. 369, no. 1, pp. 15-21, 2010.
[28] S. Stević, "Norm of weighted composition operators from Bloch space to $H_{\mu}^{\infty}$ on the unit ball," Ars Combinatoria, vol. 88, pp. $125-127,2008$.

[29] S. Stević, "Norms of some operators from Bergman spaces to weighted and Bloch-type spaces," Utilitas Mathematica, vol. 76, pp. 59-64, 2008.

[30] S. Stević, "Norm of weighted composition operators from $\alpha$ Bloch spaces to weighted-type spaces," Applied Mathematics and Computation, vol. 215, no. 2, pp. 818-820, 2009.

[31] S. Stević, "Norms of some operators on bounded symmetric domains," Applied Mathematics and Computation, vol. 216, no. 1, pp. 187-191, 2010.

[32] S. Stević, "Norm of an integral-type operator from Dirichlet to Bloch space on the unit disk," Utilitas Mathematica, vol. 83, pp. 301-303, 2010.

[33] W. Rudin, Real and Complex Analysis, McGraw-Hill, New York, NY, USA, 3rd edition, 1987. 


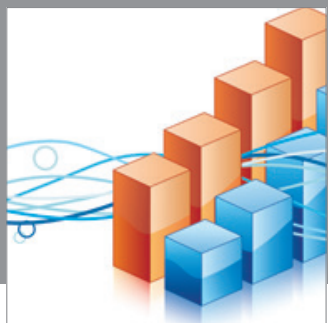

Advances in

Operations Research

mansans

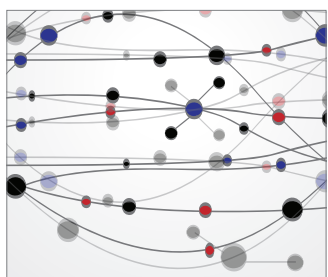

The Scientific World Journal
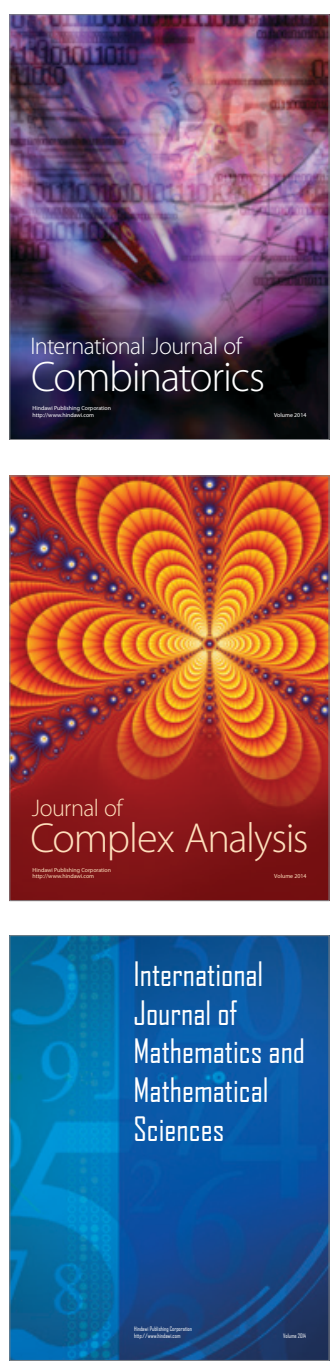
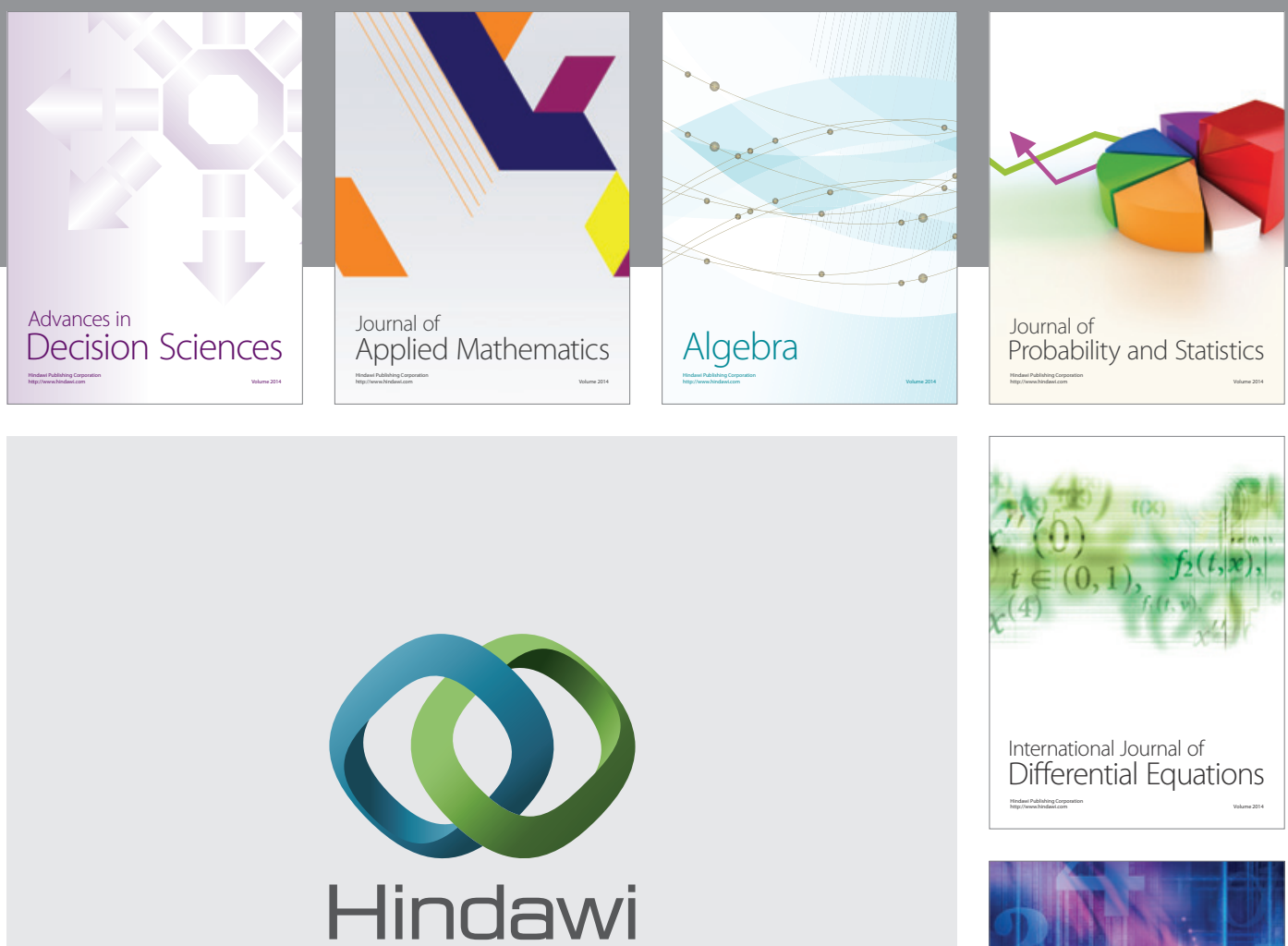

Submit your manuscripts at http://www.hindawi.com
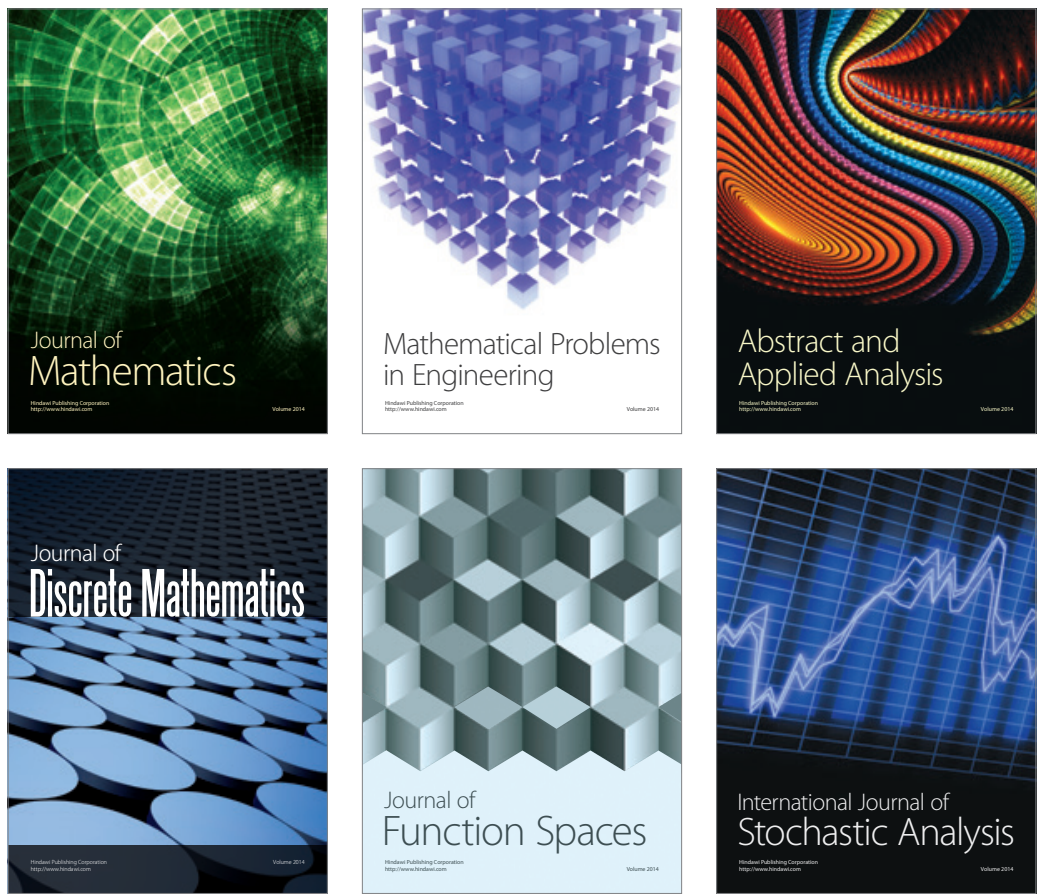

Journal of

Function Spaces

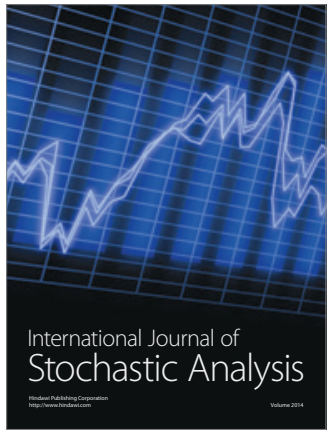

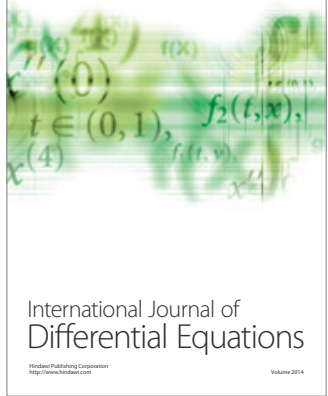
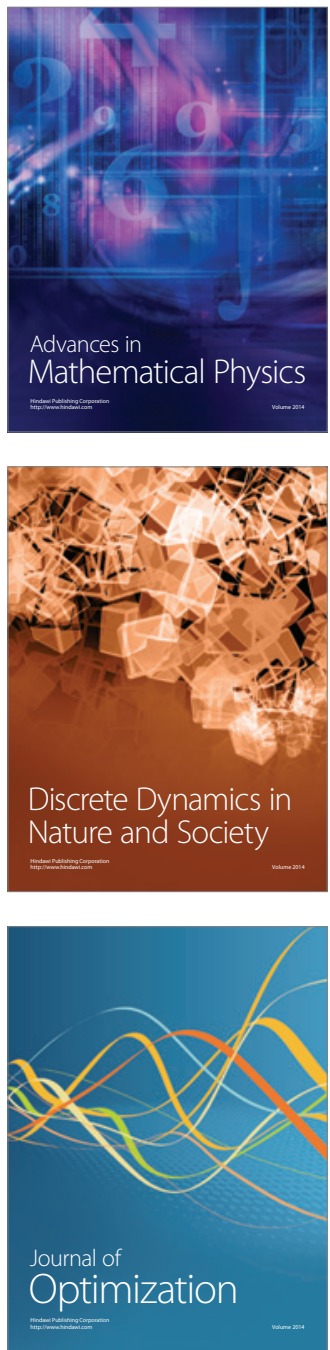